\title{
TEMPORAL ANALYSIS OF ALL HIGH-RESOLUTION MARS IMAGING PRODUCTS
}

\author{
Panagiotis Sidiropoulos*, Jan-Peter Muller
}

Imaging Group, Mullard Space Science Laboratory, University College London, Holmbury St Mary, Dorking, Surrey, RH56NT, UK p.sidiropoulos@ucl.ac.uk,j.muller@ucl.ac.uk

\section{Commission IV, WG-8}

KEY WORDS: Temporal sampling, maps of high-resolution images, Mars, statistical analysis, planetary data, metadata

\begin{abstract}
:
A meta-data analysis has been performed of high-resolution imagery that have been acquired over the last four decades from Mars. More specifically, we are interested in two independent image parameters, the time that each image was acquired and the spatial resolution with which the planetary region is mapped in the image. We are only interested in mapping changes in high-resolution images. We use two different upper thresholds to discriminate them from low-resolution images, twenty metres and a hundred metres per pixel. In order to be able to extract semantic information about the temporal and spatial distribution of high-resolution Martian imagery we adopt two grouping strategies. In the first, images are clustered according to the time period (counted in Martian Years) that they were acquired, so as to examine whether sporadic Martian phenomena can be identified (e.g. a new crater) from imagery that depict the same area in different time periods. In the second grouping, images are clustered according to the Martian season that they were acquired, so as to examine whether seasonal Martian phenomena can be identified from imagery that depict the same area during the same season. This analysis supports the hypothesis that there is sufficient coverage for both tasks, since the Martian surface has been mapped at least once in each epoch and more than twice since 2002 and for each season at least $10 \%$ of Martian surface has been mapped at least three times. The resulting maps and graphical plots will be presented will provide additional detail to this report.
\end{abstract}

\section{INTRODUCTION}

Starting from Viking Orbiter 1, launched in August 1975, several, mainly NASA, spacecraft have been sent into orbit around Mars to image its surface. Initial analyses of these early images indicated a planet with similar characteristics to the Moon pitted with craters and large volcanic and tectonic features but apparently "dead" from a geological perspective. Recently, scientific interest has shifted towards repeat highresolution imaging, which allows the identification of previously undiscovered geological phenomena and surface features as well as the examination of surface composition and geological history.

The increasing coverage of Mars orbiters, carrying highresolution cameras, allows a more comprehensive dynamic analysis of the Martian surface, i.e. the analysis of the temporal evolution of certain areas that display natural processes that happen over time. The latter can be roughly classified into two major categories; processes that happen seasonally during each and every Martian season (e.g. seasonal flows in high latitude areas (McEwen, 2011) and sporadic events that do not follow some iterative pattern (e.g. new impact craters, Byrne, 2009). In this work we conduct two temporal groupings of Mars imaging products, the first examining product distribution through time, so as to point out areas that favour the search for sporadic events, and the second product distribution per season, in order to identify areas favouring the search for periodic events.

The rest of the paper is structured as follows. Input metadata and methods are presented in the Section 2, followed by results and a sample of the maps that are going to be presented in the conference, which are given in Section 3. Future work will conclude this report.

\section{MATERIALS AND METHODOLOGY}

A Martian day (i.e. a "sol") lasts 24 hours, 39 minutes and 35 seconds and a Martian year approximately 668.6 sols (Allison, undated). Due to the fact that the orbit of Mars around the Sun is elliptical, the seasons of Mars are not of equal duration. The length of spring, summer, autumn and winter is 193.3, 178.64, 142.7 and 153.95 sols, respectively (loc.cit.). In order to be able to deal with this, it is common to count Martian time through areocentric longitude Ls, which is the relative seasonal advance of the Sun, counted in degrees. Ls ranges from $0^{\circ}$ to $359^{\circ}$, while a value equal to $0^{\circ}, 90^{\circ}, 180^{\circ}$ and $270^{\circ}$ correspond to the Mars northern hemisphere vernal equinox, summer solstice, autumnal equinox, and winter solstice, respectively (loc.cit.). While a globally accepted Martian calendar is not currently available, the vernal equinox of 11 April 1955 is usually adopted as the beginning of Mars Year 1 (MY1) (Clancy, 2000). This places $1^{\text {st }}$ January 2014 at the end of spring of MY32 and Viking Orbiter launch at MY11.

Consequently, the currently available products are drawn from a period of $\sim 20-21$ Martian years. However, imaging over that time period is discontinuous and non-uniform. Instead, analysis of the metadata demonstrate mainly episodic imaging with a gap of 9 Martian years between MY14 and MY23 that reduce the actual imaging range to $\sim 11$ Martian years overall.

In particular, six orbiter missions have conducted extensive high-resolution mapping of the Martian surface, viz. NASA's Viking Orbiter 1, Viking Orbiter 2, Mars Global Surveyor, Mars Odyssey and Mars Reconnaissance Orbiter and ESA's Mars Express. The Viking Orbiter missions were the first to perform extensive orbital mapping of the Martian surface. Viking Orbiter 1 launched on 20 August 1975 followed by Viking Orbiter 2, 20 days later (Soffen \& Synder, 1976a). Both of them reached Mars at the beginning of the northern

${ }^{*}$ Corresponding Author 
hemisphere summer of Mars Year 12 (Soffen, 1976b) and performed global mapping during MY 12-14. The resolution varied from less than 10 metres per pixel to more than $1 \mathrm{~km}$ per pixel.

After an absence of almost 17 years, the Mars Global Surveyor, with the Mars Orbiter Cameras - Narrow Angle (MOC-NA) and Wide Angle (MOC-WA) - onboard, reached Mars in September of 1997 (Albee et al., 2001), at the end of the northern hemisphere summer of Mars Year 23. The extensive imaging of the Martian surface using these two cameras continued until 3 November 2006 (Ls 130, MY 28), during which 97,097 MOC-NA and 146,591 MOC-WA images were acquired (Malin et al., 2010). MOC-NA acquired images from selected regions of interest with a spatial resolution varying from 1.5 to 12 metres per pixel while MOC-WA repeatedly covered the planet with imagery of resolution from 240 metres to 7.5 kilometres per pixel.

The next NASA orbiter to reach Mars with an imager onboard was 2001 Mars Odyssey. Mars Odyssey started acquiring imagery from 24 October 2001 (Ls 258, MY 25) and is still working at the end of MY 31 (which is the temporal limit in this work). It is thus the longest surviving orbiting imager. Images are taken with the Thermal Emission Imaging System (THEMIS) (Christensen et al., 2004), which consists of THEMIS-VIS for images and the THEMIS-IR for infrared images. The nominal target for the THEMIS-VIS was to map $60 \%$ of the Martian surface with resolution of 18 metres per pixel, but due to its longevity it has significantly surpassed this limit. It should be noted that in our statistical analysis we take into account only visible spectrum images (i.e. only THEMISVIS) and not infrared at this stage.

The only non-NASA orbiter mission that has achieved near global coverage of Martian surface up until the present-day is the High Resolution Stereo Coverage (HRSC) camera on board ESA's Mars Express (Scholten, 2005). HRSC is the only 3D mapping camera launched to date, designed to achieve global coverage of the Mars surface with 12.5-25 metres per pixel resolution, which is being used to generate a $3 \mathrm{D}$ model of the surface with spatial resolution of $50 \mathrm{~m}-100 \mathrm{~m}$. Mars Express entered Mars orbit on 25 December 2003 (Ls 322, MY 26) and continues to image until the end of MY 31.

The last orbiter mission under study is the NASA Mars Reconnaissance Orbiter, which was launched on 12 August 2005 and reached Mars at 10 March 2006 (Ls 23, MY 28). Onboard are two high-resolution cameras, the High Resolution Imaging Science Experiment (HiRISE) (McEwen et al., 2007) and the Context Camera (CTX) (Malin et al., 2007). The former acquires images with $0.25-0.5$ metres per pixel resolution in selected regions of interest, while the latter images with 6-12 metres per pixel resolution at a global scale. Both of them continue to operate until the end of the time period that is under examination.

It can be deduced from the above meta-data that imaging cameras have pixel resolutions that vary from 25 centimetres per pixel for HiRISE to thousands of metres per pixel. In order to impose some homogeneity in our analysis and to focus on change related to surface features, all images with resolution coarser than $100 \mathrm{~m}$ per pixel are ignored. This leads to ignoring all MOC-WA as well as many Viking Orbiter products. The rest of the images are grouped into two classes. The former, named "F"ine, consists of images with resolution finer than 20 metres per pixel and includes a sub-set of THEMIS-VIS, HRSC,
Viking Orbiter 1 and Viking Orbiter 2 images, and all MOCNA images, CTX and HiRISE images. On the other hand, the latter, named "C"oarse, consists of the rest of the images with 20-100 metres resolution.

$\mathrm{F}$ and $\mathrm{C}$ images are further decomposed into 2 further groups, using a different clustering criterion. More specifically, $F$ images (C images) that were acquired at MY12 to MY14, MY23 to MY25, MY26 to MY28 and MY29 to MY 31 are grouped into sub-classes F12, F23, F26 and F29 (C12, C23, $\mathrm{C} 26$ and $\mathrm{C} 29$ ), respectively, while $\mathrm{F}$ images (C images) that were acquired during Martian spring, summer, autumn, winter are grouped into sub-classes F1, F2, F3 and F4 (C1, C2, C3 and C4), respectively.

\section{ANALYSIS RESULTS}

The overall spatial surface coverage that each camera has achieved can be found in Table 1. This Table demonstrates that the coverage of orbiter cameras can be classified into two categories, the former focusing on imaging specific Martian surface regions with very high-resolution (MOC-NA, HiRISE) whilst the latter is focused on imaging the entire surface of Mars, at the expense of very high resolution (THEMIS-VIS, HRSC, CTX). From analysis of these three cameras, HRSC has achieved the most complete mapping of the Martian surface whilst CTX has the finest resolution mapping. Furthermore, the longevity of THEMIS-VIS results in $81.8 \%$ coverage (compared to $60 \%$, which was the nominal goal), thus providing an additional global Mars imagery source. Overall, these statistics demonstrate that for a large part of the Martian surface area these 3 different imaging sources contain most of the images with resolution finer than $100 \mathrm{~m}$ per pixel. MOC-NA and HiRISE provide additional sources for regions of increased scientific interest, whilst Viking Orbiter allows the analysis of the long-term temporal evolution of approximately $20 \%$ of the Martian surface for the last 20 Martian Years.

\begin{tabular}{|c|c|}
\hline Camera & Coverage \\
\hline VO1 + VO2 & $19.11 \%$ \\
\hline MOC-NA & $5.12 \%$ \\
\hline THEMIS-VIS & $81.8 \%$ \\
\hline HRSC & $97.63 \%$ \\
\hline CTX & $78.48 \%$ \\
\hline HiRISE & $1.39 \%$ \\
\hline
\end{tabular}

Table 1: Martian surface coverage for each orbiter camera. Duplicates are not included. Images with resolution coarser than 100 metres per pixel are ignored

Subsequently, we ignore the different sources and extract a histogram of the repeat image coverage of the Martian surface, using two distinct resolution thresholds, 20 metres and 100 metres per pixel (Table 2). From this analysis we have estimated that the median coverage for an area is 6 images for resolution finer than 100 metres and 4 images for resolution finer than 20 metres. Moreover, only $19 \%$ of the Martian surface has been mapped less than 3 times with resolution finer than 20 metres per pixel, a statistic that falls to $5.29 \%$ if images with resolution between 100 metres and 20 metres per pixel are taken into account. The above statistics demonstrate that it appears to be generally feasible to perform an extensive comparison of the different imagery corresponding to the same area. 


\begin{tabular}{|c|c|c|}
\hline Repeat Coverage & Resolution $<\mathbf{2 0 m}$ & Resolution $<\mathbf{1 0 0 m}$ \\
\hline Not Covered & $0.98 \%$ & $0.12 \%$ \\
\hline$\geq 1$ & $99.02 \%$ & $99.88 \%$ \\
\hline$\geq 3$ & $81.05 \%$ & $94.71 \%$ \\
\hline$\geq 5$ & $43.56 \%$ & $73.09 \%$ \\
\hline$\geq 7$ & $16.97 \%$ & $43.25 \%$ \\
\hline$\geq 20$ & $4.24 \%$ & $14.78 \%$ \\
\hline 20 & $0.45 \%$ & $2.35 \%$ \\
\hline
\end{tabular}

matter of fact, while $\mathrm{NH}$ autumn and winter are up to $30 \%$ shorter than $\mathrm{NH}$ spring and summer, larger surface coverage has been achieved during these seasons. Additionally, it appears that there is repeat coverage for the same season (i.e. at least three images with resolution finer than 20 metres per pixel) for $9.13 \%-17.32 \%$ of the Martian surface. Thus, it becomes apparent that a substantial area of Mars can be examined to analyse the characteristics of currently unknown seasonal phenomena, based on distinct and common features in images of the same corresponding area taken during the same season.

Table 2: Repeat Martian surface coverage with resolution finer than $100 \mathrm{~m}$ (AlI), exclusively finer than $20 \mathrm{~m}$ (Fine) and exclusively coarser than $20 \mathrm{~m}$ and finer than $100 \mathrm{~m}$.

This kind of comparison would benefit from images that are taken at different time periods (for events that do not follow some iterative pattern) and from images taken during the same season (for periodical events). The corresponding grouping results are summarized in Tables 3 and 4, the former corresponding to different Martian Year periods, and the latter to different Martian Seasons

Table 3 shows the Martian surface that was covered on the four examined MY periods, i.e. MY 12-14 (starting 19 December 1975 and ending 9 August 1981), MY 23-25 (starting 27 August 1996 and ending 18 April 2002), MY 26-28 (starting 19 April 2002 and ending 8 December 2007), and MY 29-31 (starting 9 December 2007 and ending 31 July 2013).

\begin{tabular}{|c|c|c|}
\hline MY Period & $\begin{array}{c}\text { Coverage } \\
(\text { Res }<20 \mathrm{~m})\end{array}$ & $\begin{array}{c}\text { Coverage } \\
(100 \mathrm{~m}<\text { Res }<20 \mathrm{~m})\end{array}$ \\
\hline $12-14$ & $0.31 \%(0.001 \%)$ & $18.89 \%(1.49 \%)$ \\
\hline $23-25$ & $3.79 \%(0.07 \%)$ & $1.32 \%(0 \%)$ \\
\hline $26-28$ & $89.79 \%(45.29 \%)$ & $67.57 \%(17.02 \%)$ \\
\hline $29-31$ & $84.65 \%(27.99 \%)$ & $33.71 \%(2.11 \%)$ \\
\hline
\end{tabular}

Table 3: Martian surface coverage statistics during different time periods. In parentheses is reported the surface percentage that was mapped more than 3 times over the same time period.

It becomes apparent from Table 3 that Mars was imaged during a minimum of two different time periods, in MY26-28 (4/0212/07) and MY 29-31 (12/07-7/13), respectively. During the first period, two different "global" Mars sets can be found, the first including the $89.79 \%$ of the surface with high resolution (i.e. finer than 20 metres per pixel) and the second including the $67.57 \%$ of the surface with medium resolution (finer than 100 metres but coarser than 20 metres per pixel). On the contrary, during the last time period, the medium resolution mapping seems to be gradually abandoned, since only $33.71 \%$ of the surface has been mapped at such a resolution.

Another conclusion that can be derived from this Table is that before MY 26 there is only a sparse mapping of Mars with high resolution (3.79\% in MY $23-25$ and $0.31 \%$ in MY 12-14) and intermediate mapping with medium resolution $(1.32 \%$ in $\mathrm{MY}$ $23-25$ and $18.89 \%$ in MY 12-14). This shows that temporal analysis can be straightforwardly conducted only for the last (earth) decade, while it will be necessary to analyse imagery with different resolution for older epochs.

Finally, in Table 4, statistics of the coverage at different Martian seasons are reported. A first conclusion that can be derived is that the coverage is independent from the season duration. As a

\begin{tabular}{|c|c|c|}
\hline Season & $\begin{array}{c}\text { Coverage } \\
(\text { Res }<20 \mathrm{~m})\end{array}$ & $\begin{array}{c}\text { Coverage } \\
(100 \mathrm{~m}<\text { Res }<20 \mathrm{~m})\end{array}$ \\
\hline $\begin{array}{c}\text { Spring } \\
\left(0^{\circ}<\text { Ls }<90^{\circ}\right)\end{array}$ & $59.88 \%(11 \%)$ & $42.77 \%(4.62 \%)$ \\
\hline $\begin{array}{c}\text { Summer } \\
\left(90^{\circ}<\text { Ls }<180^{\circ}\right)\end{array}$ & $50.51 \%(9.13 \%)$ & $27.07 \%(2.98 \%)$ \\
\hline $\begin{array}{c}\text { Autumn } \\
\left(180^{\circ}<\text { Ls }<270^{\circ}\right)\end{array}$ & $67.58 \%(13.29 \%)$ & $35.78 \%(3.96 \%)$ \\
\hline $\begin{array}{c}\text { Winter } \\
\left(270^{\circ}<\text { Ls }<360^{\circ}\right)\end{array}$ & $69.72 \%(17.32 \%)$ & $36.11 \%(4.68 \%)$ \\
\hline
\end{tabular}

Table 4: Martian surface global coverage statistics for different (Martian) seasons. In parentheses is reported the surface percentage that was mapped more than 3 times at the same season.

It can also be observed that a large area of the Martian surface remains un-covered for each season. This can be explained by the fact that, just like in Earth, the northern (southern) latitude (polar) zones of Mars are mostly at dark during the winter (or summer). Since we are currently dealing with images in the visible spectrum, this feature determines a pattern in which images are acquired according to the season.

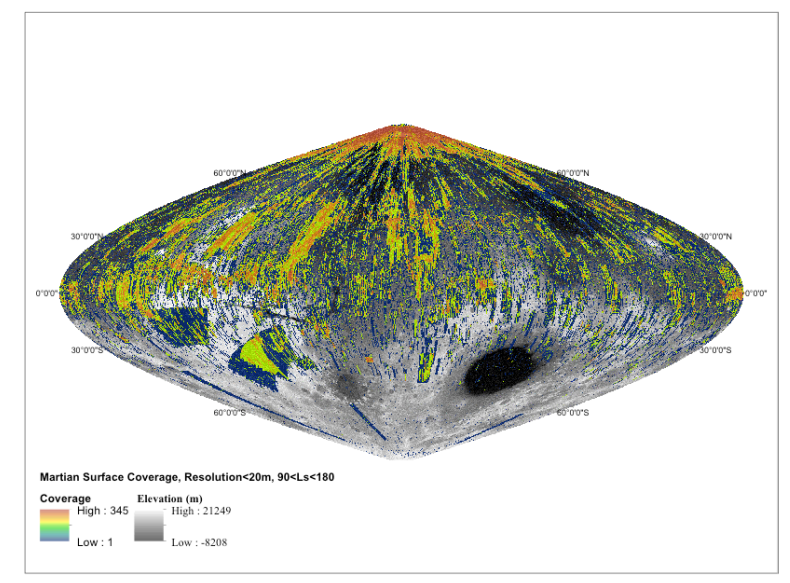

Figure 1: Martian surface coverage during north hemisphere summer $\left(90^{\circ}<\mathrm{Ls}<180^{\circ}\right)$ from 1977-2013.

These conclusions are further demonstrated in Figures 1 and 2, which demonstrate a global coverage map of Mars for $\mathrm{NH}$ summer $\left(90^{\circ}<\mathrm{Ls}<180^{\circ}\right)$ and winter $\left(270^{\circ}<\mathrm{Ls}<360^{\circ}\right)$. In both maps only images with resolution finer that 20 metres per pixel are taken into account. Furthermore, "reddish" areas depict regions that are repeatedly imaged (up to 345 times for summer and 196 times for winter) whilst "bluish" areas regions that have sparse repeat coverage. The background MOLA image can be only be observed in areas that are never imaged during the corresponding season. Figures 1 and 2 show evidence that during $\mathrm{NH}$ summer mostly north hemisphere regions are mapped whilst during $\mathrm{NH}$ winter mostly southern hemispheric 
regions are mapped. Moreover, the high latitudinal coverage gap of summer is explained by the high concentration of images to the North Pole, which is an area of extensive scientific interest.

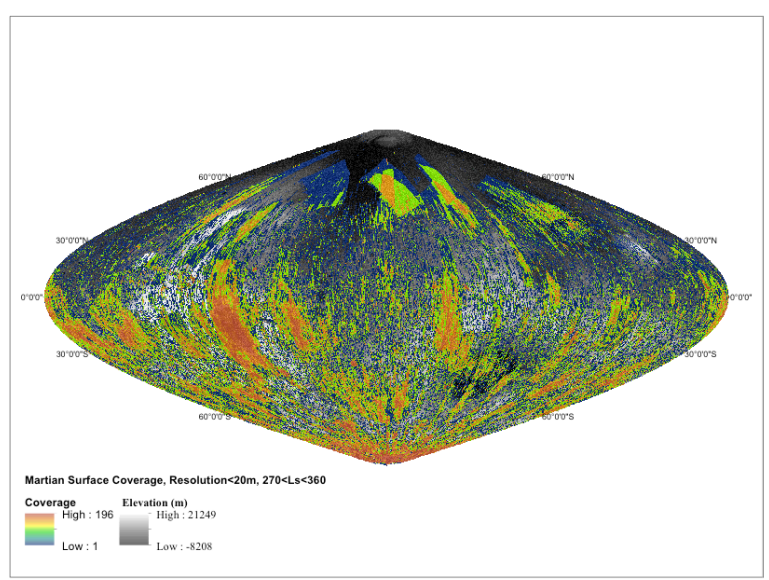

Figure 2: Martian surface coverage during north hemisphere winter $\left(270^{\circ}<\mathrm{Ls}<360^{\circ}\right)$ from $1977-2013$.

The above Figures are examples from only 2 of the 24 maps (namely, maps F2 and F4) that will be presented during the ISPRS TCIV symposium and are omitted here due to lack of space. From these 24 maps, 16 correspond to the previously discussed sub-classes, i.e. they would be a plot of the number of times each Martian region was imaged at the sub-class context. For example, a pixel with a value 12 in an F2 map would correspond to an area that was mapped 12 times during $\mathrm{NH}$ summer with resolution finer than 20 metres per pixel. The map raster grid is $500 \mathrm{~m} \times 500 \mathrm{~m}$ using a sinusoidal projection. Additionally, since polar regions are of major interest 8 further availability maps will be presented for "N"orth and "S"outh polar regions using the season-based decomposition and $\mathrm{F}$ image group (i.e. N1, N2, N3, N4, S1, S2, S3 and S4). In this case stereographic projection will be used and a $100 \mathrm{mx} 100 \mathrm{~m}$ raster.

\section{CONCLUSIONS}

In this paper we have conducted a temporal sampling analysis of all the high-resolution Mars imaging products that are available from MY 12 (i.e. Viking Orbiter missions in 1977) until the end of MY 31, which corresponds to 31 July 2013. Through this analysis we have shown that for a substantial part of Mars multiple high-resolution imaging products acquired at different time and in the same Martian season can be found. This allows us, within the Martian geological context, to assess the potential of change detection for each geographic region on the Martian surface, which will be the focus of our future research. A similar analysis is planned for the Moon in future.

\section{REFERENCES}

Albee A. L., Arvidson R. E., Palluconi F., and Thorpe T., Overview of the Mars Global Surveyor mission, 2001, Journal of Geophysical Research, 106 (E10), pp. 23291-23316

Allison, M. Technical notes on Mars Solar Time, www.giss.nasa.gov/tools/mars24/help/notes.html

Byrne, S., C. Dundas, M. Kennedy, M. Mellon, A. McEwen, S. Cull, I. Daubar, D. Shean, K. Seelos, S. Murchie, B. Cantor, R.
Arvidson, K. Edgett, A. Reufer, N. Thomas, T. Harrison, L. Posiolova and F. Seelos, 2009, Distribution of mid-latitude ground ice on Mars from new impact craters, Science, 325 (5948), pp. 1674-1676

Christensen P. R., Jakosky B. M., Kieffer H. H., Malin M. C., McSween H. Y. Jr, Nealson K, Mehall G. L., Silverman S. H., Ferry S., Caplinger M. and Ravine M., 2004, The Thermal Emission Imaging System (THEMIS) for the Mars 2001 Odyssey Mission, Space Science Reviews, 110 (1), pp. 85-130

Clancy, R.T., B. J. Sandor, M. J. Wolff, P. R. Christensen, M. D. Smith, J. C. Pearl, B. J. Conrath and R. J. Wilson, 2000, An intercomparison of ground-based millimetre, MGS TES, and Viking atmospheric temperature measurements: Seasonal and interannual variability of temperatures and dust loading in the global Mars atmosphere, Journal of Geophysical Research: Planets, 105 (E4), pp. 9553-9571

Malin M. C., Edgett K. S., Cantor B. A., Caplinger M. A., Danielson E., Jensen E. H., Ravine M. A., Sandoval J. L. and Supulver K. D., 2010, An overview of the 1985-2006 Mars Orbiter Camera science investigation, Mars, 5, pp. 1-60

McEwen, A., L. Ojha, C. Dundas, S. Mattson, S. Byrne, J. Wray, S. Cull, S. Murchie, N. Thomas and V. Gulick, 2011, Seasonal Flows on Warm Martian Slopes, Science, 333 (6043), pp. $740-743$

McEwen A., Eliason E., Bergstrom J., Bridges N., Hansen C., Delamere W., Grant J., Gulick V., Herkenhoff K., Keszthelyi L., Kirk R., Mellon M., Squyres S., Thomas N. and Weitz C., 2007, Mars reconnaissance orbiter's high resolution imaging science experiment (hirise). Journal of Geophysical Research: Planets, $112(5)$

Malin M. C., Bell J. F., Cantor B. A., Caplinger M. A., Calvin W. M., Clancy R. T., Edgett K. S., Edwards L., Haberle R. M., James P. B., Lee S. W., Ravine M. A., Thomas P. C., and Wolff M. J., 2007, Context camera investigation on board the mars reconnaissance orbiter, Journal of Geophysical Research: Planets, 112 (E5)

Scholten F., Gwinner K., Roatsch T., Matz K. -D., Wählisch M., Giese B., Oberst J., Jaumann R., Neukum G., and the HRSC Co-Investigator Team, 2005, Mars Express HRSC data processing-Methods and operational aspects. Photogrammetric Engine Remote Sensing, 71, pp. 1143-1152

Soffen, G. A., and C. W. Snyder, 1976a, First Viking mission to Mars, Science, 193, pp. 759-766

Soffen, G. A. 1976b, Scientific results of the Viking mission, Science, 194, No. 4271, pp. 1274-1276, 1976.

\section{Acknowledgements:}

The research leading to these results has received funding from the STFC "MSSL Consolidated Grant" ST/K000977/1 and partial support from the European Union's Seventh Framework Programme (FP7/2007-2013) under iMars grant agreement $n^{\circ}$ 607379 